\title{
Glycogen Storage Disease: An Unusual Presentation
}

\author{
S AFROZA $^{\mathrm{a}}$, MA ROUF ${ }^{\mathrm{b}}$, MM HAQUE $^{\mathrm{c}}$
}

\begin{abstract}
Summary:
Glycogen storage disease(GSD) are a group of disorders caused by lack of enzymes involved in glycogen synthesis or breakdown with buildup of glycogen in tissue. The incidence of glycogen storage disease (GSD) is not uncommon in paediatric population. We report the case of a twelve year old boy of glycogen storage disease who presented with gradual abdominal distension since his two years of age and two episodes of epistaxis. There was no history of jaundice, blood transfusion, convulsion, contact with TB patient, travelling to malaria or kala azar endemic
\end{abstract}

\section{Introduction:}

A number of genetic syndromes have been identified that results from some metabolic defect in the synthesis or catabolism of glycogen. The best understood and most important category includes the glycogen storage disease (glycogenoses or dextrinosis) ${ }^{1}$. Glycogen storage diseases are a group of autosomal recessive disorders in which defect in glycogen metabolism typically cause an accumulation of glycogen in the tissue, hence the name ${ }^{2}$. Glucose is the principal substrate of energy metabolism and a continuous source of glucose from dietary intake, gluconeogenesis and glycogenolysis maintain normal blood glucose levels ${ }^{2}$. The breakdown of hepatic glycogen provides the rapid release of glucose, which maintains a constant blood glucose concentration ${ }^{2}$. Glycogen is also the primary stored energy source in muscle, providing glucose for muscle activity during exercise ${ }^{2}$. The disorders of $\mathrm{GSD}_{\mathrm{s}}$ result from deficiencies of specific enzymes or transport proteins in the pathway of glycogen metabolism resulting in abnormal deposition in various organs and tissues $^{2}$. According to a study in British Columbia, approximately 2.3 children per 100,000 births (one in 43,000 ) have some form of glycogen storage disease ${ }^{3}$.

a. Prof. Syeda Afroza, Professor \& Head, Dept of Paediatrics, SSMC \& Mitford Hospital, Dhaka.

b. Dr. MA Rouf, Associate Professor of Paediatrics, SSMC \& Mitford Hospital, Dhaka.

c. Dr. MM Haque, IMO, Dept of Paediatrics, SSMC \& Mitford Hospital, Dhaka.

Address of Correspondence: Prof. Syeda Afroza, Head, Dept of paediatrics, SSMC \& Mitford Hospital.

Received: 17 December, 2013 Accepted: 25 November, 2014 zone or deterioration of school performance. On examination, he was mildly pale, stunted and had gross hepatosplenomegaly. The single positive lab investigation in this case was increased triglyceride and the final diagnosis was made by liver biopsy. After diagnosis, treatment was provided by counseling and appropriate feeding advice. This case is being reported because this type of presentation with gross hepatosplenomegaly without a single episode of convulsion is unusual in glycogen storage disease, and the age of presentation is also late.

(J Banagladesh Coll Phys Surg 2015; 33: 48-51)

In the United States, they are estimated to occur in one per 20,000-25,000 births ${ }^{3}$. A Dutch study estimated it to be one in $40,000{ }^{3}$. The overall GSD incidence is estimated at 1 case per 20,000-43,000 live births ${ }^{4}$. TypeÉ, occurs due to deficiency of glucose-6-phosphatase, is the most common (25\% of all GSD) ${ }^{4}$. On the basis of specific enzyme deficiencies and the resultant clinical pictures, glycogenoses have traditionally been divided into a dozen or so syndromes designated by roman numerals ${ }^{5}$. An another popular pathophysiological classification is also available which describes glycogenoses as having-hepatic type, myopathic type \& miscellaneous type ${ }^{5}$. The hepatic form being the most common and classically present as doll like face, failure to thrive, early morning hypoglycemia, protuberant abdomen, hepatomegaly \& renomegaly, whereas muscle weakness or elevated muscle enzyme is the main symptom of myopathic GSDs ${ }^{6}$. Easy bruising and epistaxis are common and are associated with a prolonged bleeding time as a result of impaired platelet aggregation and adhesion ${ }^{2}$. The biochemical hallmarks are hypoglycemia, lactic acidosis, hyperuricemia, and hyperlipidemia ${ }^{2}$. Edgar Von Gierke described the first patient with glycogen storage disease type 1 (GSD1) in 1929, hence the disease was named after him ${ }^{6}$. The diagnosis of GSDs is based on clinical symptoms, biochemical markers \& definitely confirmed by liver biopsy with electron microscopy and assay of enzyme activity in the tissue and/or specific gene testing ${ }^{7}$. Treatment depends on the type of GSD and the symptoms. The modalities include dietary manipulation to prevent hypoglycemia, allopurinol to reduce uric acid nephropathy, correction of acute metabolic problems, 
liver transplantation and avoidance of strenuous exercise (myopathic type $)^{8}$. Early diagnosis and early initiation of an effective treatment improve the outcome of the disease, but it is not known if all long-term complications can be avoided by good metabolic control. Some early treated patients who are now adults still developed hepatic adenomas and proteinuria ${ }^{8}$.

\section{Case Report:}

Nasim a twelve year old boy, fourth issue of a consanguineous parents got admitted into paediatric unit I of SSMC \& $\mathrm{MH}$ with the complaints of gradual distension of abdomen since 2 years of age. His family sought medical treatment from different primary and secondary level hospitals \& qualified private practitioners for this illness but no cure was achieved. On query, his mother stated that there were 2 episodes of epistaxis during his illness. There was no history of jaundice, blood transfusion, convulsion, contact with TB patient, travelling to malaria or kala azar endemic zone or deterioration of school performance. Nasim's one elder sister and one elder brother died of similar illness at the age of 25 years and 5 years respectively; his another sister and parents are healthy. On examination, he was mildly pale, anicteric, having normal vital signs, and had no lymphadenopathy. Anthropometry revealed stunting (HAZ below $3^{\text {rd }}$ centile) and BMI was 16.31. Systemic examination revealed normal findings except gross hepatosplenomegaly $(\mathrm{L}-9 \mathrm{~cm}, \mathrm{~S}-17 \mathrm{~cm})$. Considering the possibility of Wilson disease, 24 hours urinary copper $(<20 \mu \mathrm{g} / 24 \mathrm{hr})$, serum ceruloplasmin (32mg/dl) and slit lamp examination of eye were done but reports were normal. CBC- nonspecific findings, SGPT-30U/L,
S.bilirubin-0.2mg/dl, Prothrombin time (C-14, Pt-21, INR-1.71). USG of hepatobiliary system revealed hepatosplenomegaly with liver having uniform parenchymal echotexture. Hb electrophoresis revealed normal (HbA-97.7\%, $\mathrm{HbA}_{2}-2.3 \%$ ) results. Though the presentation was not classical, keeping in mind the suspicion of storage disease, liver biopsy was planned. While preparing for biopsy, his other test reports were available like fasting lipid profile showing increased triglyceride (208mg/dl) \& FBS (5.8mmol/l), S.uric acid$2.3 \mathrm{mg} / \mathrm{dl}$, S.lactate-13.4mg/dl, CPK-18U/L, MT negative \& ICT for Kala azar was also negative. Liver biopsy report revealed- "hepatocytes are swollen and

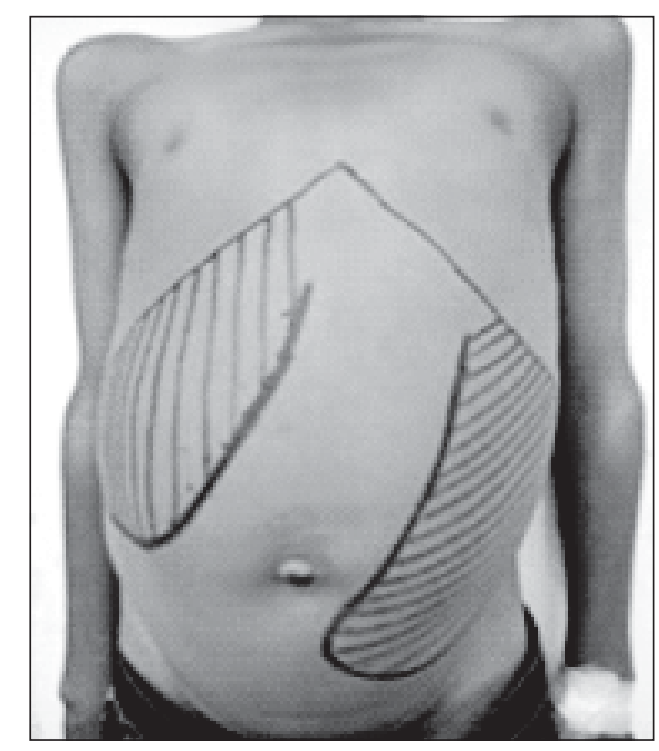

Fig.-1: Gross hepatosplenomegaly

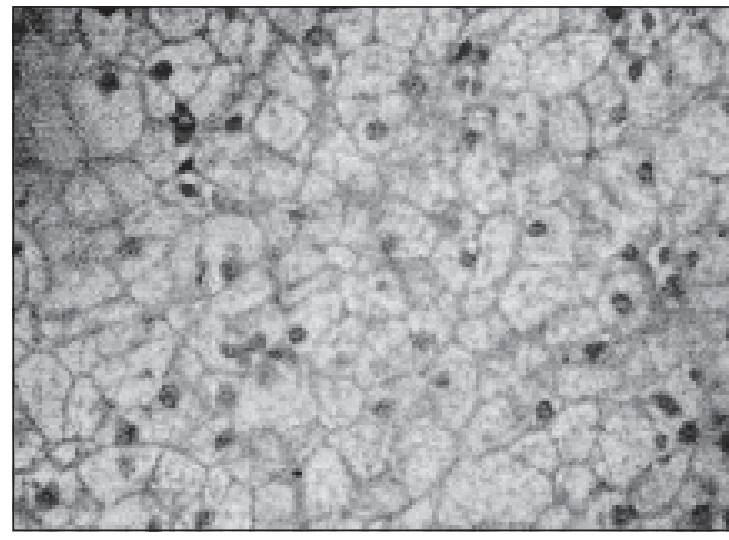

$3 a$

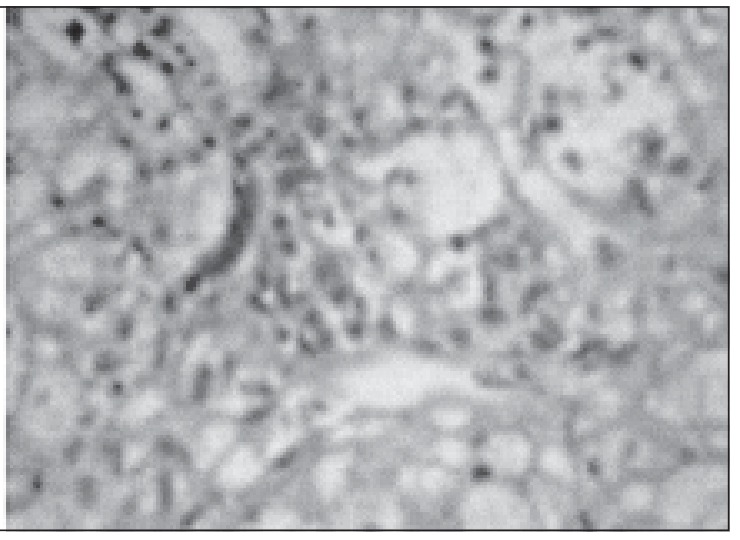

$3 b$

Fig.-2 (a, b): Photomicrograph of section of hepatic tissue reveals hepatocytes are swollen with clear cytoplasm with central nucli. The hepatic architexture is distorted. 
show clear cytoplasm with central nucli. The hepatic architexture is distorted. PAS stain shows evidence of glycogen storage disease". Finally, in this case, the diagnosis of Glycogen Storage Disease was made depending on histopathology. Due to lack of facilities, enzyme assay and gene detection were not possible and hence the specific type of GSD could not be diagnosed. After diagnosis, we counseled the parents about the nature of the disease, treatment options \& prognosis. We gave the child inj.vit-K (konakion) as PT was prolonged which became normal after treatment. We also advised him to take measured amount of uncooked cornstarch which slowly releases glucose in the intestinal lumen and then discharged. Now, Nasim is under regular follow up at the dept. of Paediatrics, SSMC.

\section{Discussion:}

Glycogen storage disease (GSD, also glycogenosis or dextrinosis) is the result of defects in the processing of glycogen synthesis or breakdown within muscles, liver, and other cell types ${ }^{9}$. GSD has two classes of cause: genetic and acquired. Genetic GSD is caused by any inborn error of metabolism (genetically defective enzymes) involved in these processes. In livestock, acquired GSD is caused by intoxication with the alkaloid castanospermie ${ }^{9}$. About 1 in 20,000 people can have GSD. GSDIa is more common than GSDIb, accounting for 80 percent of all GSDI cases ${ }^{10}$. GSD is passed down through families and occurs because of an inherited defective gene from both parents ${ }^{11}$. Mutations in two genes, G6PC and SLC37A4, cause GSDI. G6PC gene mutations cause GSDIa, and SLC37A4 gene mutations cause GSDIb. Mutations in the G6PC and SLC37A4 genes prevent the effective breakdown of glucose 6-phosphate. Glucose 6phosphate that is not broken down to glucose is converted to glycogen and fat so it can be stored within cells. Too much glycogen and fat stored within a cell can be toxic. This buildup damages organs and tissues throughout the body, particularly the liver and kidneys, leading to the signs and symptoms of GSDI. The enzymes defect that result in GSD type I, II \& III are glucose-6-phosphatase, acid alpha-glucosidase, \& glycogen debranching enzyme respectively ${ }^{10}$.

The GSD that mainly affect the liver are types I, III, IV, and VI. The glycogen storage diseases that mainly affect muscles are types V and VII. Type II affects nearly all organs, including the heart ${ }^{12}$. The symptoms of a glycogen storage disease depend on its type. The following are the common symptoms: Low blood sugar, enlarged liver, slow growth, bleeding, muscle cramps ${ }^{12}$. In the present case the features do not uniquely resemble any particular type and the most amazing issue was no incidence of hypoglycemia till date. Some of the clinical presentations of this case like delayed onset, unexplained organomegaly and epistaxis are also present in other storage diseases like Gaucher Disease but the biopsy findings ultimately excluded the possibility of others. Prolonged PT in our patient does not match with the diagnosis of GSD. As SGPT and sonographic liver findings were normal so we considered it to be lab error.

More than half of patients with GSD I tend to develop hepatocellular adenomas during the second decade of life. Ineffective treatment of the disease increases the incidence of hepatic adenomas that may undergo malignant transformation. Malignant transformation of hepatocellular adenomas into hepatocellular carcinoma has been reported in 10 cases $^{13}$. Four cases of GSD III in Inuit children from northern Quebec and the Baffin Region of eastern Nunavut in the last decade, Of note, there was no consanguinity of the parents of these children, nor was there any known shared pedigree between the 4 cases. This is comparable to the distribution of cases in other countries with an increased incidence of the disease, such as Japan. Interestingly, linkage studies tracing mitochondrial DNA sequences suggest that the circumpolar Inuit are originally of Asian progeny $^{14}$.

The diagnostic modalities are mainly blood biochemistries, liver biopsy and enzyme assay. Prenatal diagnosis has been made by fetal liver biopsy at 1822 weeks of gestation, but no fetal treatment has been proposed ${ }^{7}$. Prenatal diagnosis is possible with fetal DNA obtained by chorionic villus sampling when a fetus is known to be at risk. None of the glycogenoses are currently detected by standard or extended newborn screening ${ }^{7}$. For the types of glycogen storage disease that can be treated, patient must carefully follow a special diet- frequent high carbohydrate meals during the day, cornstarch, continuous night time feeding; allopurinol to counteract hyperuricemia. If liver failure occurs, liver transplantation is the only option. The present patient needs feeding advice and counseling. 


\section{References:}

1. Di Mauro S. Biochemistry and molecular genetics of human glycogenosis: an overview.2nd ed. St. Louis: Mosby; 1995.

2. Priya S, Kishnani, Yuan-Tsong C. Defects in Metabolism of Carbohydrates. In: Kliegman RM, Stanton BF, St Geme JW, editors. Nelson Textbook of Pediatrics.19th ed. Philadelphia: WB Ssunders Company; 2011. p 492-509.

3. Boundless Learning.Galactosemia and Glycogen Storage Disease;[accessed 3rd June 2013].Available from:https:// www.boundless.com/physiology/nutrition-metabolism-andtemperature-regulation/clinical-cases-metabolism/ galactosemia-and-glycogen-storage-disease/

4. Ozen H. Glycogen storage diseases: new perspectives. World J Gastroenterol. 2007; 13(18): 2541-53.

5. Chen Y-T. Glycogen storage disease. In:. Fauci AS, Braunwald E, Isselbacher KJ, Wilson JD, Martin JB, Kasper DL, et al, editors. Harrison's Principles of Internal Medicine.7th ed. New York: Mc-Graw-Hill; 1998. p 2176.

6. Moses SW. Historical highlights and unsolved problems in glycogen storage disease type 1. Eur J Pediatr. 2002; 161(1): 2-9.

7. Wikipedia.Glycogen storage disease type; [ accessed 6th June 2013].Available from: http://en.wikip edia.org/wiki/ Glycogen_storage_disease_type

8. Sondra F,Jane F.Glycogen Storage Diseases Treatment;[accessed $10^{\text {th }}$ November 2013]. Available from:http://www.thirdage.com/hc/c/glycogen-storagediseases-treatment

9. Wikipedia.Glycogen storage disease;[accessed 6 th June 2013].Available from:. https://en.wikipedia.org /wiki/ Glycogen_storage_disease/

10. US national library of medicine; 2010. Glycogen storage disease type-I; [updated 23 Sep 2013; accessed 30 May 2013]. Available from: http://ghr.nlm.nih.gov/conditi-On/glycogenstorage-diseasi-type-i .

11. Ohio: Cincinnati Children's Hospital Medical Center; 1999.Glycogen storage disease; [updated 17 Nov 2012; accessed 30 May 2013].Available from:https:// cincinnatichildrens.org/health/g/gsd

12. CChildren's Hospital of Pittsburgh of UPMC. Glycogen storage disease; [updated 14 Aug 2008; accessed 5 July 2013]. Available from: http://www.chp.edu/CHP/glycogen+storage+ diseasesAv.

13. Endoh A, Inoue N, Katoh T, Nakamura T, Sugimura H, Ohzeki T. A Case of Glycogen Storage Disease IA With Multiple Hepatic Tumors Managed by Transcatheter Arterial Embolization and an Acarbose Diet. Journal of Pediatric Gastroenterology \& Nutrition. 2001; 33: 333-336.

14. Bonatto SL, Salzano FM. A single and early migration for the peopling of the America supported by mitochondrial DNA sequence data. Proc Natl Acad Sci U S A. 1997; 94(5): 1866-71. 